\title{
Dopaminergic Substantia Nigra Neurons Project Topographically Organized to the Subventricular Zone and Stimulate Precursor Cell Proliferation in Aged Primates
}

\author{
Nils Freundlieb, ${ }^{1,2 \star}$ Chantal François, ${ }^{2 \star}$ Dominique Tandé, ${ }^{2}$ Wolfgang H. Oertel, ${ }^{1}$ Etienne C. Hirsch, ${ }^{2}$ and \\ Günter U. Höglinger ${ }^{1}$ \\ ${ }^{1}$ Experimental Neurology, Philipps University, D-35033 Marburg, Germany, and 'Institut National de la Santé et de la Recherche Médicale, Unité Mixte de \\ Recherche 679, Experimental Neurology and Therapeutics, Hôpital de la Salpetrière, Université Pierre et Marie Curie Paris 6, F-75651 Paris, France
}

\begin{abstract}
The subventricular zone of the adult primate brain contains neural stem cells that can produce new neurons. Endogenous neurogenesis might therefore be used to replace lost neurons in neurodegenerative diseases. This would require, however, a precise understanding of the molecular regulation of stem cell proliferation and differentiation in vivo. Several regulatory factors, including dopamine, have been identified in rodents, but none in primates. We have, therefore, studied the origin and function of the dopaminergic innervation of the subventricular zone in nonhuman primates. Tracing experiments in three macaques revealed a topographically organized projection from the substantia nigra pars compacta $(\mathrm{SNpc})$, but not the adjacent retrorubral field, to the subventricular zone: the anteromedial SNpc projects to the anteroventral subventricular zone, the posterolateral $\mathrm{SNpc}$ to the posterodorsal subventricular zone. Double immunolabeling for tyrosine hydroxylase and BrdU (5-bromo-2'deoxyuridine) incorporated into the DNA of proliferating cells showed that dopaminergic fibers approach proliferating cells in the subventricular zone. We investigated the effect of this nigro-subventricular projection on cell proliferation in six aged macaques, because the rate of neurogenesis differs between young adult and aged primates and because neurodegenerative diseases mainly affect aged humans. Three macaques were treated with MPTP (1-methyl-4-phenyl-1,2,3,6tetrahydropyridine) to decrease dopaminergic innervation of the subventricular zone. A significant decrease in the number of PCNA ${ }^{+}$ (proliferating cell nuclear antigen-positive) proliferating cells $(-44 \%)$ and $\mathrm{PSA}^{-\mathrm{NCAM}^{+}}$(polysialylated neural cell adhesion moleculepositive) neuroblasts $(-59 \%)$ was found in the denervated regions of the subventricular zone, suggesting that an intact dopaminergic nigro-subventricular innervation is crucial for sustained neurogenesis in aged primates.
\end{abstract}

Key words: nonhuman primate; aging; dopamine; adult neurogenesis; neural precursor cells; substantia nigra; Parkinson's disease

\section{Introduction}

The subventricular zone (SVZ) in the adult primate brain contains neural stem cells that have the potential to produce new neurons, astrocytes, and oligodendrocytes (Kornack and Rakic, 2001; Sanai et al., 2004). Under physiological conditions, most of the cells born in the SVZ migrate to the olfactory bulb, where they differentiate and integrate as interneurons (Kornack and Rakic, 2001). Under some pathological conditions, such as cerebral ischemia, Huntington's disease, or demyelination, SVZ-derived neural cells appear to contribute to brain repair (Arvidsson et al., 2002; Picard-Riera et al., 2002; Curtis et al., 2003). Controlled stimulation of these spontaneous repair processes might be an elegant way to treat neurodegenerative diseases. It is important

\footnotetext{
Received Nov. 11, 2005; revised Jan. 10, 2006; accepted Jan. 14, 2006.

This work was supported by the German Federal Ministry of Education and Research Network "Stem Cells in PD," the Peter Hofmann Research Project, a Boehringer Ingelheim research fellowship to N.F., the Förderverein Neurologie Marburg, the French Network "Neural Stem Cells for Cell Therapy in PD and MS," the Institut National de la Santé et de la Recherche Médicale, and Fondation de l'Avenir. We thank Merle Ruberg for revising this manuscript.

${ }^{*}$ N.F. and C.F. contributed equally to this work.

Correspondence should be addressed to Günter U. Höglinger, Experimental Neurology, Philipps University, D-35033 Marburg, Germany. E-mail: guenter.hoeglinger@med.uni-marburg.de. DOI:10.1523/JNEUROSCI.4859-05.2006

Copyright $\odot 2006$ Society for Neuroscience $\quad$ 0270-6474/06/262321-05\$15.00/0
}

therefore to understand how the proliferation, migration, and differentiation of endogenous neural stem and precursor cells are regulated. However, up to now, nothing has been known about the molecular signals that govern neurogenesis in adult primates.

The neurotransmitter dopamine is an important factor to stimulate precursor cell proliferation in the SVZ of adult rodents (Baker et al., 2004; Coronas et al., 2004; Höglinger et al., 2004; Van Kampen et al., 2004; Winner et al., 2006). Consistently, we found a reduced rate of cell proliferation in the SVZ of patients with Parkinson's disease, a condition characterized by forebrain dopamine depletion secondary to degeneration of the dopaminergic neurons in the substantia nigra pars compacta $(\mathrm{SNpc})$ (Höglinger et al., 2004). The dopaminergic regulation of SVZ precursor cell proliferation appears therefore to be conserved in humans, but the present evidence is weak, because it is difficult to control for all potentially confounding factors such as concomitant disease or medication that might influence the evaluation of neurogenesis in studies on human postmortem material. Furthermore, it is not known whether the dopaminergic innervation of the SVZ actually degenerates in Parkinson's disease, because not all dopaminergic cell populations are equally vulnerable to the disease process (Hirsch et al., 1988). We have therefore examined in adult primates whether, first, there is an axonal projection 
from the SNpc to the SVZ and whether, second, a destruction of the dopaminergic neurons in the $\mathrm{SNpc}$ results in reduced precursor cell proliferation in the SVZ.

\section{Materials and Methods}

Animals. All experiments were performed in accordance with the European Communities Council Directive of 1986 (86/609/EEC). The monkeys were housed in individual cages under standard conditions $(12 \mathrm{~h}$ light cycles; $23^{\circ} \mathrm{C} ; 50 \%$ humidity). They had not been used previously for experimentation. For tracing studies, we used three Cercopithecus aethiops monkeys, 4-6 years of age, weighing 5.5-6.5 kg. To study the effect of dopamine depletion, we used six aged macaques (Macaca mulatta), 20-25 years of age, weighing $8-15 \mathrm{~kg}$. Two young macaques ( $\mathrm{Ma}$ caca fascicularis), 4-6 years of age, weighing $3.5-4 \mathrm{~kg}$, were used to compare the SVZ of young adult and aged macaques. Both species used (Ceropithecus, Macaca) belong to the same Old World monkey subfamily (Cercopithecine) and both have a comparable body size and brain development.

BrdU labeling. We injected unlesioned aged macaques under anesthesia (10 mg ketamine/kg body weight, i.m.) with the thymidine analog 5-bromo-2' deoxyuridine (BrdU) (Sigma, St. Louis, MO) to label proliferating cells. So that infrequently dividing precursors would be labeled, $\mathrm{BrdU}$ (40 mg BrdU/kg body weight, $5 \mathrm{mg} / \mathrm{ml}$ in $0.9 \% \mathrm{NaCl}$ with $7 \mathrm{~mm}$ $\mathrm{NaOH}$, i.p.) was injected 10 times at 2 d intervals over a 3 week period (cumulative dose per animal, $400 \mathrm{mg}$ BrdU/kg body weight). Animals were killed 3 weeks after the last BrdU injection to allow the majority of restricted precursors to migrate out of the SVZ (Kornack and Rakic, 2001). The $\mathrm{BrdU}^{+}$cells remaining in the SVZ are therefore likely to be upstream elements in the neural cell lineage (Höglinger et al., 2004).

Tracing studies. To label axonal projections from midbrain dopaminergic neurons, three $C$. aethiops monkeys were deeply anesthetized by i.m. injections of $10 \mathrm{mg}$ of ketamine and $0.1 \mathrm{ml}$ of atropine per kilogram of body weight, and kept anesthetized by inhalation of $1 \%$ fluothane in $50 \%$ nitrogen protoxide $50 \%$ oxygen. The anterograde tracer biotin dextran amine (BDA) (Sigma; $10 \%$ in $0.01 \mathrm{M}$ PBS) was injected iontophoretically in $6.8 \mu \mathrm{A}$ pulses lasting $7 \mathrm{~s}$, at $7 \mathrm{~s}$ intervals over a $20 \mathrm{~min}$ period into the posterolateral or anteromedial part of the SNpc or into the retrorubral field. One monkey was used for each condition. The stereotaxic injections were guided by radiological visualization of anatomical landmarks (ventricles, anterior and posterior commissure) (Percheron et al., 1986). Ten to $12 \mathrm{~d}$ later, the animals were anesthetized, placed in a stereotaxic apparatus to introduce a vertical cannula into each cerebral hemisphere perpendicular to the plane passing through the two ventricular landmarks, and killed by an overdose of anesthetic and transcardial perfusion with $0.5 \mathrm{~L}$ of heparinized saline $(0.9 \% \mathrm{NaCl})$, followed by $5 \mathrm{~L}$ of fixative (4\% paraformaldehyde, $2.5 \%$ sucrose in saline) and $1 \mathrm{~L}$ of icecold $0.1 \mathrm{M}$ PBS containing $5 \%$ sucrose. The brains were removed from the skull and immersed in 10\% sucrose in PBS for $1 \mathrm{~d}$ and in $20 \%$ sucrose for $2 \mathrm{~d}$, and cut into $50 \mu \mathrm{m}$ sections on a freezing microtome using the vertical cannulas as guide. Sections were stored in $0.1 \mathrm{M}$ PBS containing $0.2 \%$ sodium azide.

MPTP treatment. To deplete brain dopamine, three aged macaques received intramuscular injections of the dopaminergic neurotoxin 1-methyl-4-phenyl-1,2,3,6-tetrahydropyridine (MPTP) (Sigma; dissolved in saline; $0.4 \mathrm{mg} / \mathrm{kg}$ body weight) at $2-3 \mathrm{~d}$ intervals under anesthesia (ketamine, $10 \mathrm{mg} / \mathrm{kg}$ body weight). The monkeys were examined behaviorally every other day. MPTP injections were repeated until a stable parkinsonian syndrome was present (four to six MPTP injections per animal; cumulative dose, $1.2-2 \mathrm{mg} / \mathrm{kg}$ ). Three aged control macaques received saline injections. The animals were killed 5 weeks after the last MPTP or saline injection with a lethal dose of ketamine and perfused transcardially with $2 \mathrm{~L}$ of saline. Brains were removed, cut midsagitally in two hemispheres, and divided into tissue blocks. Blocks from one hemisphere were frozen rapidly for biochemical analysis. Blocks from the other hemisphere were postfixed for $3 \mathrm{~d}$ in $4 \%$ paraformaldehyde in $0.1 \mathrm{M}$ PBS, immersed in $10 \%$ sucrose in PBS for $1 \mathrm{~d}$ and $20 \%$ sucrose for $2 \mathrm{~d}$, and cut in $40 \mu \mathrm{m}$ sections on a freezing microtome.

Immunohistochemistry. Free-floating sections were incubated in $50 \%$ ethanol and $0.3 \%$ hydrogen peroxide in PBS $\left(20^{\circ} \mathrm{C} ; 30 \mathrm{~min}\right)$ to inhibit endogenous peroxidases, in PBS containing $10 \%$ normal goat serum and $0.3 \%$ Triton X-100 to block nonspecific binding sites $\left(20^{\circ} \mathrm{C} ; 30 \mathrm{~min}\right)$, and then in the following antibodies $\left(4^{\circ} \mathrm{C} ; 2 \mathrm{~d}\right)$ : mouse IgM antipolysialylated neural cell adhesion molecule (PSA-NCAM) (1:400; Chemicon, Temecula, CA), rabbit anti-tyrosine hydroxylase (TH) (1: 500; Pel-Freez, Compiegne, France), rabbit anti-dopamine $\beta$-hydroxylase (1:500; Chemicon), rabbit anti-dopamine transporter (DAT) (1:500; Chemicon), rat anti-BrdU (1:200; ImmunologicalsDirect, Oxfordshire, UK), and mouse anti-proliferating cell nuclear antigen (PCNA) (1:500; DakoCytomation, High Wycombe, UK; Glostrup, Denmark). Samples were pretreated with $2 \mathrm{~N} \mathrm{HCl}\left(37^{\circ} \mathrm{C} ; 30 \mathrm{~min}\right)$ and rinsed in $0.1 \mathrm{M}$ boric acid for BrdU detection and in $70 \%$ ethanol $\left(-20^{\circ} \mathrm{C} ; 30\right.$ min) for PCNA detection. The antibodies were visualized by peroxidase histochemistry with diaminobenzidine (DAB) as substrate (Vector Laboratories, Burlingame, CA) or with fluorescent secondary antibodies (FITC- or Cy3-conjugated goat anti-rat or rabbit IgG; 1/200; Jackson ImmunoResearch, West Grove, PA). Nuclei were stained with methyl green or 4',6'-diamidino-2-phenylindole (DAPI).

Tracer visualization. Free-floating sections were incubated with an avidin-biotin complex (ABC) (Elite; Vector Laboratories) diluted 1:100 in 0.1 м PBS containing $1 \%$ Triton X-100 $\left(20^{\circ} \mathrm{C} ; 24 \mathrm{~h}\right)$. After washing in Tris- $\mathrm{HCl}$ buffer, the $\mathrm{ABC}$ incubation was repeated. The BDA tracer was then visualized by incubation in a solution containing $0.06 \%$ hydrogen peroxide, $0.2 \%$ nickel ammonium sulfate, and $0.05 \% \mathrm{DAB}$. The reaction was stopped in Tris buffer.

Image analysis. Cell counts were performed on four regularly spaced coronal sections along the rostrocaudal extent of the SVZ from $7 \mathrm{~mm}$ anterior to $1 \mathrm{~mm}$ posterior to the anterior commissure and along the dorsoventral extent from the corpus callosum to the vena thalamostriata (Martin and Bowden, 1996), using a semiautomatic stereology system (ExploraNova Mercator). The density of $\mathrm{TH}^{+}$immunoreactivity was quantified by determining the optical density under bright-field illumination.

Statistics. Data are reported as the mean \pm SEM. Normal parametric data were compared by a two-sided, unpaired $t$ test. A value of $p<0.05$ was considered to be statistically significant.

\section{Results \\ The SVZ of old primates}

Most studies of the SVZ in primates have been performed with young adult animals. Because most neurodegenerative diseases occur in older humans, we compared the SVZ of two young adult macaques ( $4-5$ years) and three old macaques (20-25 years). Cells immunoreactive for PSA-NCAM, a marker of immature neuroblasts, were present in the SVZ of both young and old macaques (Fig. $1 A$ ). On sections stained with cresyl violet to visualize nuclei, the SVZ of both young and aged macaques was readily distinguished medially from the layer of ependymal cells with their rounded nuclei and laterally from the heterogeneous cell populations of the striatum (Fig. 1A) (Kornack and Rakic, 2001; Höglinger et al., 2004; Sanai et al., 2004). Both the diameter of the SVZ and the number of cells it contained were markedly reduced in old macaques compared with the young adults throughout the entire anteroposterior extent of the structure, whether cresyl violet-stained nuclei or PSA-NCAM-positive cells were used as markers (Fig. 1A). Therefore, to mimic the situation in neurodegenerative disorders of aged human subjects as closely as possible, we decided to study the dopaminergic influence on the SVZ of aged rather than young macaques.

\section{Dopaminergic fibers contact proliferating cells in the primate SVZ}

To analyze the spatial relationship between dopaminergic fibers and precursor cells in the SVZ of untreated aged macaques, we examined $\mathrm{TH}^{+}$catecholaminergic fibers and $\mathrm{BrdU}^{+}$proliferat- 
A
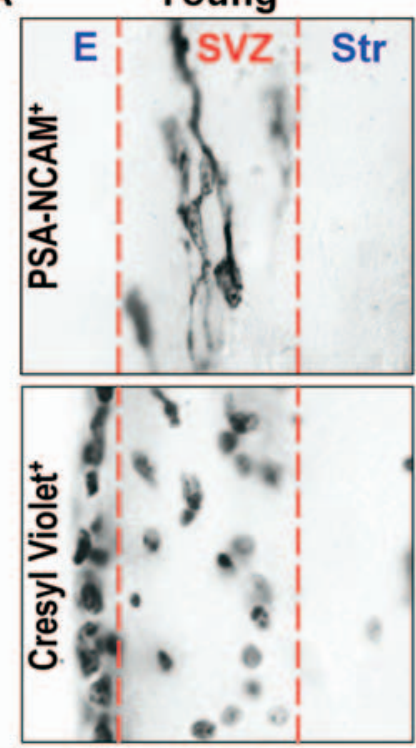

B

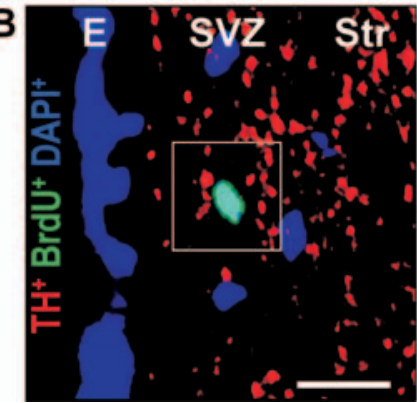

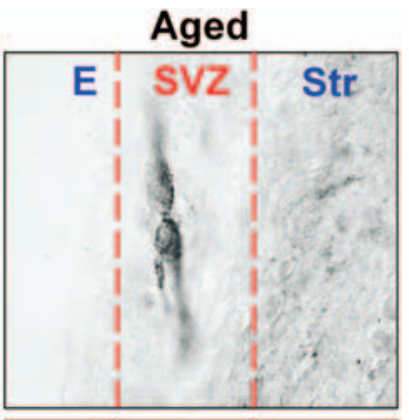
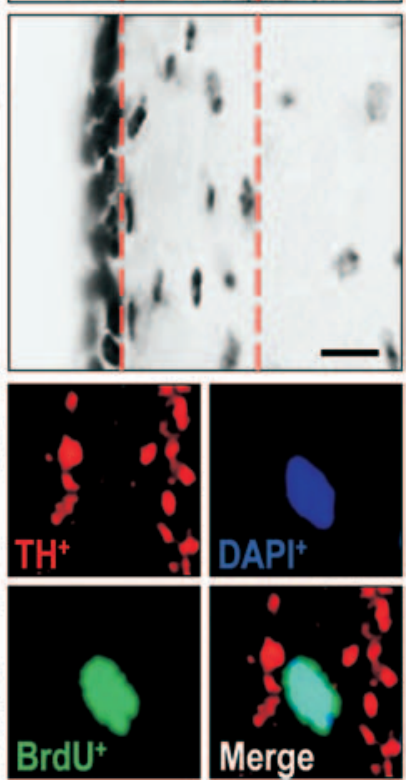

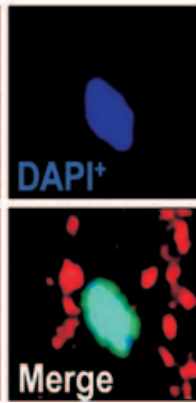

Figure 1. The SVZ of aged macaques. $A$, The number of PSA-NCAM ${ }^{+}$migrating neuroblasts was reduced in the SVZ of aged ( $20-25$ years) compared with young adult ( $4-5$ years) macaques. The SVZ, distinguished from the ependyma (E) and the striatum (Str) on the basis of the morphology and distribution of cresyl violet-stained nuclei, was smaller in aged animals. Scale $\mathrm{bar}, 20 \mu \mathrm{m} . \boldsymbol{B}$, In the SVZ of an aged macaque, fibers immunoreactive for the catecholaminergic marker TH (red) were found in close vicinity to proliferating cells, identified by immunostaining of BrdU incorporated into nuclear DNA (green). Nuclei are counterstained with DAPI (blue). The figure shows an overview and the detail of the boxed area taken on a confocal microscope. Scale bar, $20 \mu \mathrm{m}$

ing cells in the SVZ. $\mathrm{TH}^{+}$catecholaminergic fibers were in close vicinity to $\mathrm{BrdU}^{+}$SVZ cells (Fig. $1 B$ ). The fibers were dopaminergic, because they were labeled with an antibody against the dopamine transporter, but not the noradrenergic marker dopamine- $\beta$-hydroxylase.

The SNpc projects topographically to the SVZ

To determine the origin of the dopaminergic fibers in the SVZ, we stereotaxically injected the anterogradely transported axonal tracer BDA into the posterolateral part of the SNpc in one monkey and anteromedial part of the SNpc in another (Fig. $2 A$ ). The distribution of $\mathrm{BDA}^{+}$axons in the three subdivisions of the striatum (caudate nucleus, putamen, nucleus accumbens) was analyzed on serial sections (Fig. $2 B$ ). In agreement with previous reports (Lynd-Balta and Haber, 1994a,b), the nigrostriatal projection was topographically organized. The anteromedial SNpc projected preferentially to the anteroventral striatum, targeting primarily the caudate nucleus and the nucleus accumbens. The posterolateral SNpc projected preferentially to the posterodorsal striatum, targeting primarily the putamen.

The topographical organization was similar in the SVZ be-

\section{SN anteromedial SN posterolateral}
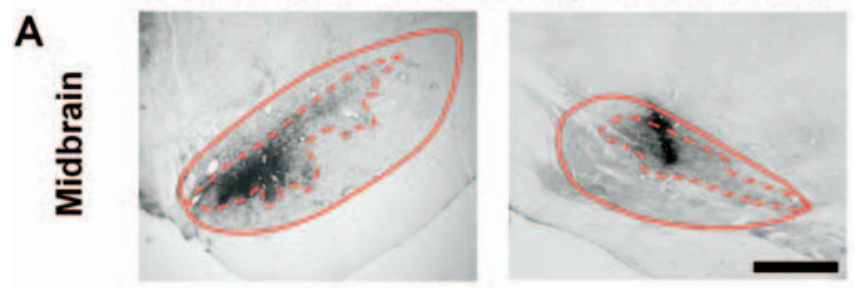

\section{B}
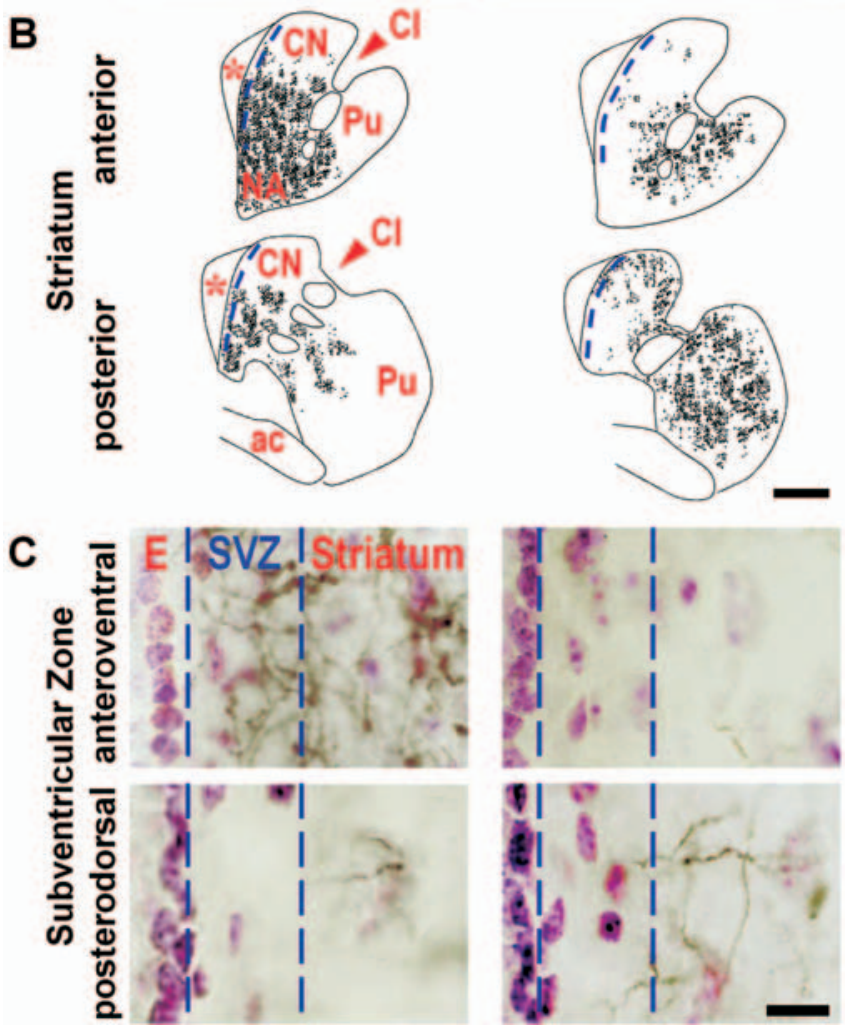

Figure 2. Topographical organization of the dopaminergic projection from the substantia nigra (SN) pars compacta to the subventricular zone (SVZ) in adult primates. The anterograde tracer BDA was injected stereotaxically into the anteromedial (first column) or posterolateral (second column) SN pars compacta. $\boldsymbol{A}$, The injection sites in the midbrain were verified histologically. The solid line delimits the $\mathrm{SN}$ as a whole, and the broken line delimits the dopaminergic SN pars compacta. Scale bar, $2 \mathrm{~mm}$. B, Schematic drawing of the distribution of the BDAlabeled axons on coronal sections of the striatum at an anterior and a more posterior level $(+4$ and $+0 \mathrm{~mm}$ from the anterior commissure). A blue broken line indicates the location of the SVZ. *, Lateral ventricle; $\mathrm{CN}$, caudate nucleus; $\mathrm{Cl}$, capsula interna; Pu, putamen; NA, nucleus accumbens; ac, anterior commissure. Scale bar, $2 \mathrm{~mm}$. C, Photomicrographs showing BDA-positive axons (brown) in the anteroventral and posterodorsal SVZ. Nuclei are counterstained with cresyl violet to distinguish the SVZ from the ependyma (E) and the striatum. Scale bar, $10 \mu \mathrm{m}$.

tween the ependymal layer covering the lateral ventricles and the striatum. The anteromedial SNpc projected preferentially to the anteroventral SVZ, the posterolateral SNpc to the posterodorsal SVZ (Fig. 2C).

To control for the specificity of this dopaminergic projection, we injected BDA into the dopaminergic retrorubral field of a third monkey. The retrorubral field is immediately caudal to the SNpc. BDA ${ }^{+}$axons were found mainly in the anterolateral and posteroventral striatum. No $\mathrm{BDA}^{+}$fibers were detected in the SVZ (data not shown).

Dopamine depletion reduces precursor cell production in the SVZ of aged primates

To study the effect of dopamine depletion on the SVZ of aged primates, three 20- to 25-year-old macaques were treated repeat- 


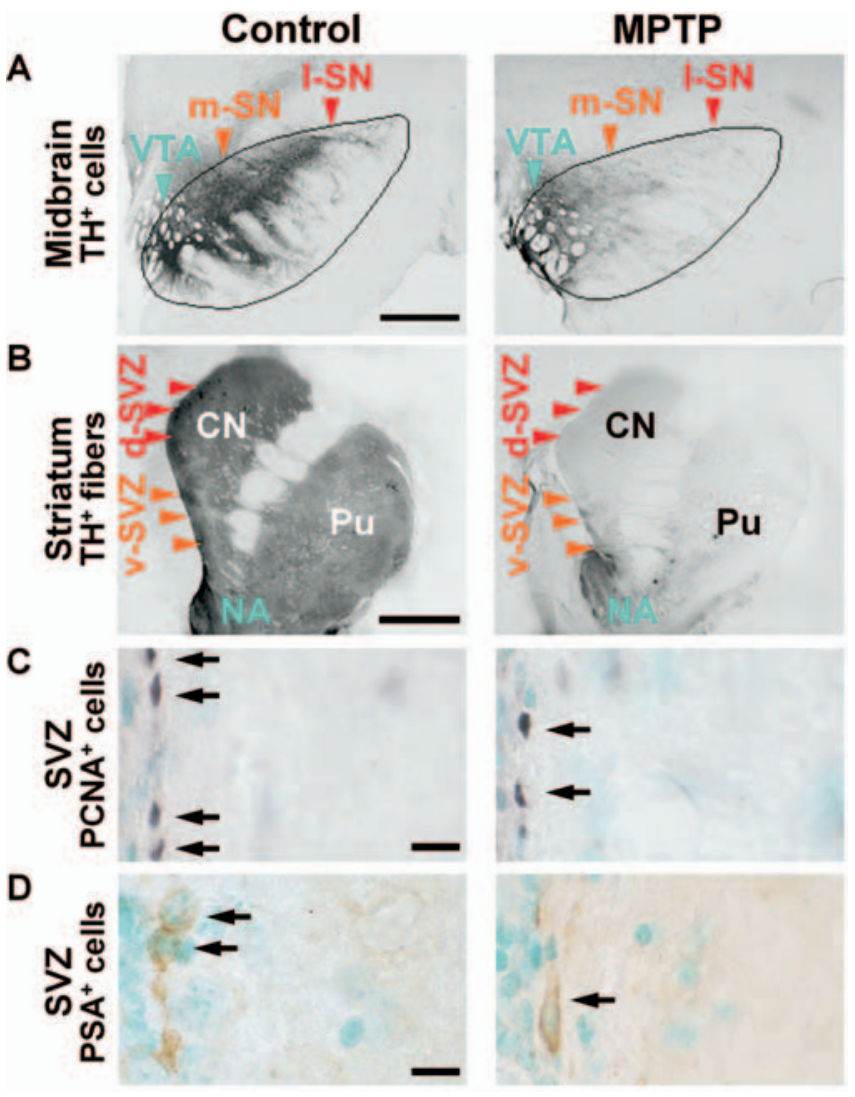

Figure 3. Dopaminergic denervation and reduction in the number of precursor cells in the SVZ of MPTP-treated aged macaques ( $20-25$ years). $\boldsymbol{A}, \boldsymbol{B}$, Dopaminergic cell bodies and fibers were visualized by immunolabeling TH in the ventral midbrain $(\boldsymbol{A})$ and striatum $(\boldsymbol{B})$ in control and MPTP-treated macaques. VTA, Ventral tegmental area; $\mathrm{SN}$, pars compacta of the substantia nigra; $m$, medial; I, lateral; $d$, dorsal; $v$, ventral; $C$, caudate nucleus; Pu, putamen; NA, nucleus accumbens. Note that degeneration of the I-SN (red) projecting to the d-SVZ (red) is severe in MPTP-treated monkeys, whereas the m-SN (orange) projecting to the v-SVZ (orange) and the VTA (blue) projecting to the NA (blue) are only moderately or mildly affected, respectively. Scale bars: $\boldsymbol{A}, \boldsymbol{B}, 2 \mathrm{~mm} . \boldsymbol{C}, \boldsymbol{D}$, Representative photomicrographs showing PCNA ${ }^{+}$proliferating cells ( $\boldsymbol{C}$, arrows) and PSA-NCAM ${ }^{+}$migrating neuroblasts ( $\boldsymbol{D}$, arrows) in the SVZ of a control and an MPTP monkey. Nuclei are counterstained with methyl green. Scale bars: $C, D, 10 \mu \mathrm{m}$.

edly with the dopamine-specific neurotoxin MPTP, as specified in Materials and Methods, and killed 5 weeks after the last injection. They were compared with three unlesioned macaques of the same age.

There was pronounced loss of $\mathrm{TH}^{+}$dopaminergic neurons in MPTP-treated macaques. Loss of $\mathrm{TH}^{+}$cell bodies was severe in the lateral SNpc, moderate in the medial SNpc, and mild in the ventral tegmental area (Fig. 3A). The anterior SNpc was more severely affected than the posterior $\mathrm{SNpc}$ (data not shown). In the caudate nucleus and putamen, there was a significant decrease in the optical density of $\mathrm{TH}^{+}$fibers (MPTP, $4.71 \pm 2.15$; control, $19.14 \pm 3.62 ; p<0.05)$, but the nucleus accumbens was not significantly affected (MPTP, $14.22 \pm 0.90$; control, $16.15 \pm$ 2.65) (Fig. $3 B$ ). In the SVZ, loss of $\mathrm{TH}^{+}$fibers was more pronounced in the dorsal SVZ receiving afferents from the anterolateral SNpc than in the ventral SVZ receiving afferents from the posteromedial SNpc (Fig. 3B).

The number of proliferating cells in the SVZ, determined by stereological analysis of $\mathrm{PCNA}^{+}$cells on regularly spaced sections spanning the entire rostrocaudal and dorsoventral extent of the structure, was significantly reduced by $44 \%$ in the MPTP monkeys compared with controls (MPTP, $44.4 \pm 9.9 / \mathrm{mm}$; con-

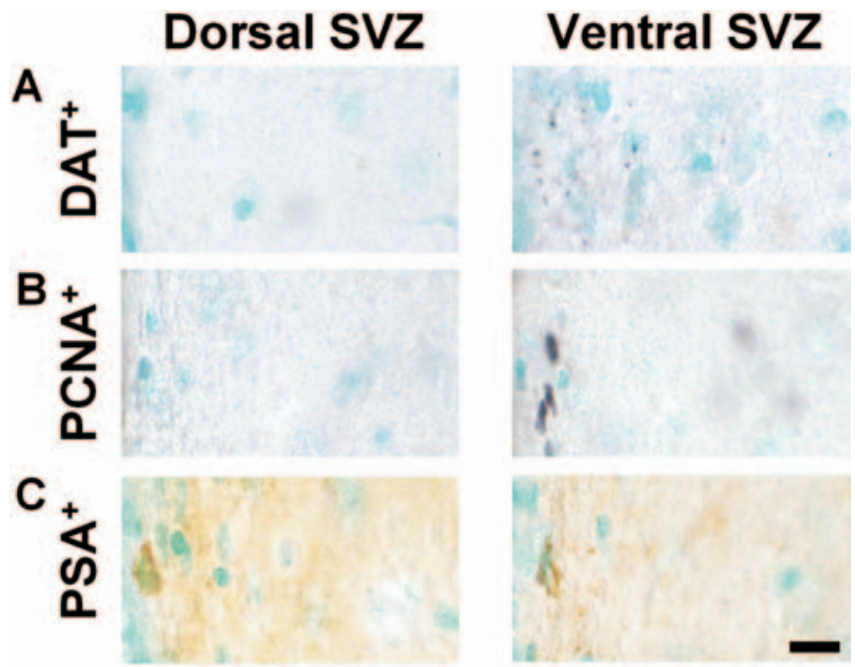

Figure 4. Cell proliferation is reduced in denervated regions of the SVZ of MPTP-treated old macaques. $\boldsymbol{A}-\boldsymbol{C}$, Representative photomicrographs of the dorsal and ventral halves of the SVZ of an MPTP-treated aged macaque stained with antibodies against the DAT to label dopaminergic fibers $(\boldsymbol{A})$, PCNA to label proliferating cells $(\boldsymbol{B})$, and PSA-NCAM to label neuroblasts $(\boldsymbol{C})$. Scale bar: (in $C A-C, 10 \mu \mathrm{m}$.

trol, $78.8 \pm 6.6 / \mathrm{mm} ; p<0.05$ ) (Fig. $3 C$ ). The number of migrating neuroblasts in the SVZ, determined by stereological analysis of PSA-NCAM ${ }^{+}$cells, was reduced by $59 \%$ in the MPTP-treated monkeys (MPTP, $13.1 \pm 1.0 / \mathrm{mm}$; control, $32.2 \pm 5.6 / \mathrm{mm} ; p<$ 0.05) (Fig. 3D).

The reduction in cell proliferation within the SVZ is correlated with dopaminergic denervation

The loss of dopaminergic $\left(\mathrm{TH}^{+}, \mathrm{DAT}^{+}\right)$fibers was virtually complete in the dorsal SVZ of MPTP-treated monkeys, whereas some dopaminergic fibers remained in the ventral SVZ (Figs. 3B, $4 A$ ). To determine whether the reduction in cell proliferation also followed a dorsoventral gradient, we analyzed the dorsal and ventral halves of the SVZ separately on each of the histological sections covering the entire rostrocaudal extent of the structure. The ratio of the number of $\mathrm{PCNA}^{+}$cells in the dorsal and ventral SVZ was $0.32 \pm 0.05$ in MPTP-treated monkeys compared with $0.78 \pm 0.15$ in controls $(p<0.05)$ (Fig. $4 B)$. This indicates a greater reduction in cell proliferation in the dorsal SVZ of MPTPtreated monkeys than in the ventral SVZ, reflecting the pattern of dopaminergic denervation. The ratio of PSA-NCAM ${ }^{+}$neuroblasts in the dorsal and ventral SVZ was similar, however, in MPTP-treated $(1.32 \pm 0.13)$ and control $(1.40 \pm 0.67)$ monkeys (Fig. 4C) indicating that dopamine depletion affected cell proliferation but did not change the distribution pattern of PSA$\mathrm{NCAM}^{+}$neuroblasts.

\section{Discussion}

We report here first evidence for the existence of a topographically organized dopaminergic projection from the SNpc to the SVZ in primates that is implicated in the regulation of adult neurogenesis.

This nigro-subventricular projection appears specific, because no axonal extensions to the SVZ from the dopaminergic retrorubral field immediately caudal to the $\mathrm{SNpc}$ was detected in our study, and because the axonal projections from the dopaminergic ventral tegmental area, medial to the $\mathrm{SNpc}$, have been shown to terminate predominantly in the nucleus accumbens (Lynd-Balta and Haber, 1994a,b), thus ventral to the SVZ. 
The similarity of the topographical organization of this projection with the organization of the nigrostriatal track, as described in this study, suggests that it might consist of axons that spread out into the SVZ from the neighboring striatum at some stage of development. This view is supported by anatomical studies demonstrating that a dopaminergic innervation arrives during embryogenesis concomitantly in both the striatum and SVZ of the developing rodent brain (Ohtani et al., 2003; Popolo et al., 2004). Functional studies demonstrated that dopaminergic $D_{2}$ like receptor stimulation increases proliferation of precursor cells from the adult rodent SVZ, whereas $\mathrm{D}_{1}$-like receptor stimulation has the opposite effect (Coronas et al., 2004; Höglinger et al., 2004), just as it is the case in the embryonic rodent SVZ (Ohtani et al., 2003; Popolo et al., 2004; Zhang et al., 2005). The net effect of dopamine, acting on both $\mathrm{D}_{1}$-like and $\mathrm{D}_{2}$-like receptors, appears to depend on the relative abundance of these receptors on precursor cells in both the embryonic and adult SVZ (Ohtani et al., 2003; Höglinger et al., 2004; Popolo et al., 2004). Together, these observations suggest an ontogenetic continuum of the dopaminergic control of precursor cell proliferation from the embryonic to the adult SVZ.

Previous work has already shown that dopamine is a potent stimulator of endogenous neural precursor cell proliferation in the adult rodent SVZ (Baker et al., 2004; Coronas et al., 2004; Höglinger et al., 2004; Van Kampen et al., 2004; Winner et al., 2006). Although dopamine has been reported to inhibit the proliferation of a population of slowly dividing stem cells in the adult rodent SVZ (Kippin et al., 2005), its stimulatory effect on the more abundant and highly proliferative precursor cells (Coronas et al., 2004; Höglinger et al., 2004) appears to predominate in vivo. In the adult rodent SVZ, dopaminergic stimulation globally increased precursor cell production (Höglinger et al., 2004; Van Kampen et al., 2004), whereas dopamine depletion globally reduced precursor cell production (Baker et al., 2004; Höglinger et al., 2004; Winner et al., 2006). In the present study, experimental dopamine depletion in aged macaques resulted in a significant decrease in cell proliferation in the SVZ, in correlation with the loss of dopaminergic innervation, and in a decrease in the number of PSA-NCAM ${ }^{+}$neuroblasts. These observations suggest that the stimulating effect of dopamine on precursor cell proliferation in the adult SVZ is functional and phylogenetically conserved from rodents to primates.

Therefore, in addition to its well known role in the regulation of mood, motivation, and movement, the SNpc also participates in the regulation of adult neurogenesis through the previously unknown dopaminergic nigro-subventricular projection described in this report. The effect of dopamine depletion on precursor cell production in primates may have important implications for human disease. Chronic dopamine depletion in humans, as occurring in Parkinson's disease, might indeed lead to a consistent reduction in precursor cell production, as it had been suggested by the results from rodent models of the disease (Baker et al., 2004; Höglinger et al., 2004; Winner et al., 2006). Thus, a chronic alteration of the precursor cell supply to the olfactory bulb might underlie olfactory dysfunction in patients with Parkinson's disease or subclinical dopamine deficiency (Berendse et al., 2001). Conversely, stimulation of dopaminergic neurotransmission by pharmacological means might prove beneficial in neurodegenerative conditions such as stroke, Huntington's disease, or multiple sclerosis, in which repair mechanisms involving SVZ-derived neural precursors have been evoked (Arvidsson et al., 2002; Picard-Riera et al., 2002; Curtis et al., 2003). The present study shows that there is an anatomical and molecular substrate permitting manipulation of endogenous neural precursor cells in the brain of aged primates. The idea that it might be used for therapeutic purposes opens exciting perspectives.

\section{References}

Arvidsson A, Collin T, Kirik D, Kokaia Z, Lindvall O (2002) Neuronal replacement from endogenous precursors in the adult brain after stroke. Nat Med 8:963-970.

Baker SA, Baker KA, Hagg T (2004) Dopaminergic nigrostriatal projections regulate neural precursor proliferation in the adult mouse subventricular zone. Eur J Neurosci 20:575-579.

Berendse HW, Booij J, Francot CM, Bergmans PL, Hijman R, Stoof JC, Wolters EC (2001) Subclinical dopaminergic dysfunction in asymptomatic Parkinson's disease patients' relatives with a decreased sense of smell. Ann Neurol 50:34-41.

Coronas V, Bantubungi K, Fombonne J, Krantic S, Schiffmann SN, Roger M (2004) Dopamine D3 receptor stimulation promotes the proliferation of cells derived from the post-natal subventricular zone. J Neurochem 91:1292-1301.

Curtis MA, Penney EB, Pearson AG, van Roon-Mom WM, Butterworth NJ, Dragunow M, Connor B, Faull RL (2003) Increased cell proliferation and neurogenesis in the adult human Huntington's disease brain. Proc Natl Acad Sci USA 100:9023-9027.

Hirsch E, Graybiel AM, Agid YA (1988) Melanized dopaminergic neurons are differentially susceptible to degeneration in Parkinson's disease. Nature 334:345-348.

Höglinger GU, Rizk P, Muriel MP, Duyckaerts C, Oertel WH, Caille I, Hirsch EC (2004) Dopamine depletion impairs precursor cell proliferation in Parkinson disease. Nat Neurosci 7:726-735.

Kippin TE, Kapur S, van der Kooy D (2005) Dopamine specifically inhibits forebrain neural stem cell proliferation, suggesting a novel effect of antipsychotic drugs. J Neurosci 25:5815-5823.

Kornack DR, Rakic P (2001) The generation, migration, and differentiation of olfactory neurons in the adult primate brain. Proc Natl Acad Sci USA 98:4752-4757.

Lynd-Balta E, Haber SN (1994a) The organization of midbrain projections to the ventral striatum in the primate. Neuroscience 59:609-623.

Lynd-Balta E, Haber SN (1994b) The organization of midbrain projections to the striatum in the primate: sensorimotor-related striatum versus ventral striatum. Neuroscience 59:625-640.

Martin RF, Bowden DM (1996) A stereotaxic template atlas of the macaque brain for digital imaging and quantitative neuroanatomy. NeuroImage 4:119-150.

Ohtani N, Goto T, Waeber C, Bhide PG (2003) Dopamine modulates cell cycle in the lateral ganglionic eminence. J Neurosci 23:2840-2850.

Percheron G, Francois C, Yelnik J (1986) Instruments and techniques for the stereotactic surgery based on the CA-CP ventricular system of coordinates in monkeys. J Neurosci Methods 17:89-99.

Picard-Riera N, Decker L, Delarasse C, Goude K, Nait-Oumesmar B, Liblau R, Pham-Dinh D, Evercooren AB (2002) Experimental autoimmune encephalomyelitis mobilizes neural progenitors from the subventricular zone to undergo oligodendrogenesis in adult mice. Proc Natl Acad Sci USA 99:13211-13216.

Popolo M, McCarthy DM, Bhide PG (2004) Influence of dopamine on precursor cell proliferation and differentiation in the embryonic mouse telencephalon. Dev Neurosci 26:229-244.

Sanai N, Tramontin AD, Quinones-Hinojosa A, Barbaro NM, Gupta N, Kunwar S, Lawton MT, McDermott MW, Parsa AT, Manuel-Garcia Verdugo J, Berger MS, Alvarez-Buylla A (2004) Unique astrocyte ribbon in adult human brain contains neural stem cells but lacks chain migration. Nature 427:740-744

Van Kampen JM, Hagg T, Robertson HA (2004) Induction of neurogenesis in the adult rat subventricular zone and neostriatum following dopamine D receptor stimulation. Eur J Neurosci 19:2377-2387.

Winner B, Geyer M, Couillard-Despres S, Aigner R, Bogdahn U, Aigner L, Kuhn G, Winkler J (2006) Striatal deafferentation increases dopaminergic neurogenesis in the adult olfactory bulb. Exp Neurol 197:113-121.

Zhang L, Bai J, Undie AS, Bergson C, Lidow MS (2005) D1 dopamine receptor regulation of the levels of the cell-cycle-controlling proteins, Cyclin D, P27 and Raf-1, in cerebral cortical precursor cells is mediated through cAMP-independent pathways. Cereb Cortex 15:74-84. 